\title{
A Novel Porcine Model of Thrombotic Myocardial Infarction with Cardiac Dysfunction for the Evaluation of Antiplatelet Agents
}

\author{
Makoto Mizuno, Yusuke Ito, Atsuhiro Sugidachi \\ Rare Disease \& LCM Laboratories, Daiichi Sankyo Co., Ltd., Japan
}

Background: Myocardial infarction (MI) is one of the leading causes of death worldwide. Dual antiplatelet therapy (DAPT) of aspirin plus P2Y12 antagonist is the first-line treatment for acute coronary syndrome. Photochemicallyinduced thrombosis (PIT) is useful for evaluating the effect of antiplatelet agents. The porcine heart is similar to the human one regarding structural and physiological characteristics. However, to our best knowledge, there are no reports of porcine MI model induced by PIT. In the present study, we tried to establish a novel porcine model of PIT-induced MI and evaluated effects of DAPT, aspirin plus prasugrel, a P2Y12 antagonist.

Methods: Thrombotic MI was induced photochemically by employing rose bengal. Male miniature pigs were divided into 3 groups; sham $(\mathrm{n}=6)$, MI $(\mathrm{n}=8)$, and DAPT $(\mathrm{n}=8)$. In the DAPT group, aspirin $(10 \mathrm{mg} / \mathrm{kg}$, p.o.) and prasugrel (1 $\mathrm{mg} / \mathrm{kg}$, p.o.) were administered $4 \mathrm{~h}$ before the photo-irradiation. Platelet aggregation, MI volume and cardiac function were evaluated $24 \mathrm{~h}$ after the photo-irradiation.

Results: Inhibition of ADP-induced platelet aggregation in the DAPT group was about 50\%, which was similar effect in clinical situations. In the sham group, no MI was observed, and end-diastolic volume (EDV), end-systolic volume (ESV), stroke volume (SV) and ejection fraction (EF) were $1.85+/-0.06 \mathrm{~mL}, 0.73+/-0.02 \mathrm{~mL}, 1.12+/-0.04 \mathrm{~mL}$ and $60.47+/-$ $0.27 \%$, respectively. PIT induced MI and the MI volume was $12.9+/-2.9 \%$ of left ventricular $(\mathrm{p}=0.0016)$. Additionally, ESV $(1.01+/-0.05 \mathrm{~mL}, \mathrm{p}=0.0006)$, SV $(0.82+/-0.04 \mathrm{~mL}, \mathrm{p}=0.0001)$ and $\mathrm{EF}(44.70+/-2.10 \%, \mathrm{p}<0.0001)$ were changed in the MI group compared to the sham group without any changes in EDV. DAPT reduced significantly MI volume $(0.1+/-0.0 \%, \mathrm{p}=0.0006)$ with amelioration of cardiac dysfunction (ESV: $0.75+/-0.02 \mathrm{~mL}, \mathrm{p}=0.0002$; SV: 1.12 +/- $0.02 \mathrm{~mL}, \mathrm{p}<0.0001$; EF: $60.21+/-0.47 \%, \mathrm{p}<0.0001)$ compared to the MI group.

Conclusions: A novel porcine model of thrombotic MI with cardiac dysfunction was established. In this model, DAPT resulted in the reduction of MI volume and amelioration of cardiac dysfunction, suggesting this porcine MI model is a potentially useful model for future research on MI and antithrombotic agents. 